\title{
Designing a novel vacuum aspiration system to decellularize large-size enthesis with preservation of physicochemical and biological properties
}

\author{
Qiang Shi ${ }^{1,2}$, Yang Chen ${ }^{1,2}$, Muzhi $\mathrm{Li}^{1,2}$, Tao Zhang ${ }^{1,2}$, Shulin Ding ${ }^{1,3}$, Yan $\mathrm{Xu}^{1,2}$, Jianzhong Hu ${ }^{1,3}$, Can Chen ${ }^{1,4}$, \\ Hongbin $\mathrm{Lu}^{1,2}$ \\ ${ }^{1}$ Department of Sports Medicine, Xiangya Hospital, Central South University, Changsha, China; ${ }^{2}$ Key Laboratory of Organ Injury, Aging and \\ Regenerative Medicine of Hunan Province, Changsha, China; ${ }^{3}$ Department of Spine Surgery, Xiangya Hospital, Central South University, Changsha, \\ China; ${ }^{4}$ Department of Orthopedics, Xiangya Hospital, Central South University, Changsha, China \\ Contributions: (I) Conception and design: H Lu, C Chen; (II) Administrative support: H Lu; (III) Provision of study materials or patients: J Hu; (IV) \\ Collection and assembly of data: Q Shi, Y Chen, M Li, T Zhang; (V) Data analysis and interpretation: Q Shi, Y Chen, S Ding, Y Xu; (VI) Manuscript \\ writing: Q Shi; (VII) Final approval of manuscript: All authors. \\ Correspondence to: Can Chen; Hongbin Lu. Xiangya Hospital, Central South University, No. 87, Xiangya Rd., Changsha, China. \\ Email: chencanwow@foxmail.com; hongbinlu@hotmail.com.
}

Background: Functional and rapid enthesis regeneration remains a challenge after arthroscopic rotator cuff $(\mathrm{RC})$ repair. Tissue-engineering a large-size biomimetic scaffold may be an adjuvant strategy to improve this clinical dilemma. Herein, we developed an optimized protocol to decellularize large-size enthesis as scaffolds for augmenting RC tear.

Methods: A novel vacuum aspiration system (VAS) was set up, which can provide a negative pressure to suck out cellular substances from tissue blocks without using chemical detergents. Large-size enthesis tissue specimens were harvested from canine infraspinatus tendon (IT) insertion, and then decellularized with an optimized protocol [freeze-thaw processing followed by nuclease digestion and phosphate buffer saline (PBS) rinsing in the custom-designed VAS], or a conventional protocol (freeze-thaw processing followed by nuclease digestion and PBS rinsing), thus fabricating two kinds of acellular enthesis matrix (AEM), namely $\mathrm{C}-\mathrm{AEM}$ and O-AEM. After that, the C-AEM and O-AEM were comparatively evaluated from the aspect of their physicochemical and biological properties.

Results: Physiochemically, the O-AEM preserved the morphologies, ingredients, and tensile properties much better than the C-AEM. Biologically, in vitro studies demonstrated that both C-AEM and O-AEM show no cytotoxicity and low immunogenicity, which could promote stem cells attachment and proliferation. Interestingly, O-AEM showed better region-specific inducibility on the interacted stem cell down osteogenic, chondrogenic and tenogenic lineages compared with C-AEM. Additionally, using a canine IT repair model, the injured enthesis patched with O-AEM showed a significant improvement compared with the injured enthesis patched with C-AEM or direct suture histologically.

Conclusions: The proposed VAS may help us fabricate large-size AEM with good physicochemical and biological properties, and this AEM may have potential clinical applications in patching large/massive RC tear.

Keywords: Tissue-engineering; acellular scaffold; enthesis regeneration; bone-tendon interface (BTI)

Submitted May 01, 2020. Accepted for publication Sep 18, 2020.

doi: 10.21037/atm-20-3661

View this article at: http://dx.doi.org/10.21037/atm-20-3661 


\section{Introduction}

Bone-tendon interface (BTI) comprises four transitional and gradient tissues: tendon, uncalcified fibrocartilage, calcified fibrocartilage, and bone (1-3). This transitional structure allows smooth transmission of forces derived from muscle contraction and minimizes the formation of stress peaks (4-6). Enthesis injuries, particularly those in the rotator cuff (RC) or anterior cruciate ligament (ACL), are prevalent conditions that often lead to disability and persistent pain (7). Regrettably, rapid and functional enthesis regeneration remains a difficulty owing to its poor capacity of self-repair (8). Current surgical approaches, which only attach the ruptured tendon and bony footprint together, are unable to recapitulate the enthesis with a transitional structure, thus resulting in a high rate of rerupture $(20-94 \%)(1,9)$.

Various tissue-engineering strategies were developed to promote enthesis regeneration with the combination of growth factors (GFs), biomaterials, stem cells, and physical stimulations (1,10-14). Previous studies attempted to engineer graded enthesis constructs as adjuncts to native tissue repair using polymer multi-phase scaffolds along with one or multiple cell types (15-19). However, synthetic scaffolds could not fully emulate the formation of the original structure and matrix components of enthesis. This led to a lack of similarity in composition and mechanical properties with respect to the extracellular matrix (ECM) of native enthesis. Thus, an ideal tissue-engineered scaffold should mimic the ECM of native enthesis.

In the past decades, a decellularization technique was developed for fabricating biomimetic scaffolds. These scaffolds showed low immunogenicity, high biocompatibility, and good biodegradability (20-22). Furthermore, they mostly preserved the inherent GFs and cytokines of the native tissue, thus forming an intricate meshwork within the tissue that interacts with the resident cells to regulate cell behaviors, such as migration, proliferation, and differentiation (20-22). Previous studies indicated that organ-specific ECM scaffolds derived from site-specific homologous tissues might be better suited for constructive tissue remodeling than no-site-specific tissue sources. Logically speaking, decellularized ECM from enthesis may provide a natural three-dimensional scaffold with tissue-specific orientations of molecules for enthesis regeneration. Fibrochondrocytes in enthesis are usually attached or deeply enclosed within the ECM
$(2,23)$. Chemical surfactant rinsing and nuclease digestion are used together to remove the cellular components and antigens of this tissue (24). Recently, Su et al. developed a protocol for large-size enthesis decellularization using $2 \%$ t-octylphenoxypolyethoxy-ethanol (Triton X-100), 3\% sodium dodecyl sulfate (SDS), and nuclease digestion (25). Such protocol properly preserved the entire natural structure and mechanical properties of the native enthesis. However, it washed away a great part of collagen and glycosaminoglycans (GAGs) in the fibrocartilage layer and took more than 10 days to remove the cellular components clearly through several complicated steps. According to prior studies, the reasons for the decrease in collagen and GAG content in the fibrocartilage may be the process of detergent treatment for a long time period and the usage of chemical surfactants $(26,27)$. Thus, a new acellular protocol for the enthesis tissue should avoid the usage of chemical detergents and shorten the decellularization time. Previous studies indicated that an acellular protocol encompassing physical methods such as tissue-trimming, freeze-thaw cycles, ultrasound, or perfusion, exhibited better decellularization with preservation of natural tissue structures and compositions than single chemical reagents (20,28-30). Recently, Liu et al. developed an acellular protocol without detergents for canine patellar tendon enthesis decellularization (31). This protocol includes the processes of tissue-trimming, freeze-thaw cycles, phosphate buffer saline (PBS) rinsing, and nuclease digestion. Unfortunately, it is still a complicated and timeinefficient protocol. The time used for the decellularization of large-size enthesis with thick fibrocartilage layer may be even longer. To improve this aspect, we decellularized large-size enthesis with freeze-thaw processing followed by nuclease digestion and PBS rinsing in a custom-design vacuum aspiration system (VAS). This VAS system provides a negative pressure environment and flows PBS rinsing on the enthesis tissue for its quick decellularization.

In this study, we aimed to conduct a comparative evaluation of the acellular effect of the conventional protocol (modified from Liu et al.'s protocol) and optimized protocol (decellularized within VAS) on large-size enthesis tissue. Additionally, the acellular enthesis matrix (AEM) prepared with both protocols were comparatively evaluated in terms of physicochemical and biological properties. We present the following article in accordance with the ARRIVE reporting checklist (available at http://dx.doi. org/10.21037/atm-20-3661). 


\section{Methods}

\section{Harvesting infraspinatus tendon (IT)-bumerus complex}

IT-humerus complex was obtained from a local slaughterhouse. After the dermis and soft tissues around the complex were removed, the humerus was sawed around the footprint of IT to acquire the IT and attached humerus bone. Excessively spongy bone was removed, leaving a sample with a size of approximately $3.0 \mathrm{~cm} \times 0.8 \mathrm{~cm} \times$ $0.5 \mathrm{~cm}$. The harvested samples were immediately rinsed with sterile $\mathrm{PBS}$ and stored at $-80^{\circ} \mathrm{C}$ before use.

\section{Preparation of AEM}

After the samples were washed in PBS to remove excess blood, the IT enthesis tissue was treated with five freezethaw cycles (one cycle consists of freezing for $2 \mathrm{~min}$ in liquid nitrogen followed by thawing for $10 \mathrm{~min}$ in $37^{\circ} \mathrm{C}$ PBS). Part of the IT enthesis tissue was decellularized with the conventional protocol, i.e., it was rinsed with PBS thrice (30 min each), digested with nuclease solution at $37^{\circ} \mathrm{C}$ for $24 \mathrm{~h}(100 \mu \mathrm{g} / \mathrm{mL}$ RNase and $150 \mathrm{IU} / \mathrm{mL}$ DNase), and finally rinsed in PBS thrice (30 min each). The other part of the IT enthesis tissue was decellularized with the optimized protocol, i.e., rinsed with flowing PBS in a self-built VAS $(0.1 \mathrm{mPa}$ negative pressure) for $1 \mathrm{~h}$ followed by nuclease solution $(100 \mu \mathrm{g} / \mathrm{mL}$ RNase and $150 \mathrm{IU} / \mathrm{mL}$ DNase) digestion at $37^{\circ} \mathrm{C}$ for $2 \mathrm{~h}$ (repeated three times). After PBS rinsing, we used a vacuum freeze-drier (FD8-5T, SIM, USA) to lyophilize these treated enthesis to obtain two types of AEM, i.e., AEM prepared with the conventional AEM protocol (C-AEM) and AEM prepared with the optimized AEM protocol (O-AEM). During decellularization, $100 \mathrm{mg} / \mathrm{mL}$ streptomycin, $100 \mathrm{U} / \mathrm{mL}$ penicillin, and $2.5 \mathrm{mg} / \mathrm{mL}$ fungizone were added into the above-mentioned solutions. Protease inhibitor ( 1 tablets $/ 300 \mathrm{~mL}, \mathrm{~S} 8820$ $20 \mathrm{TAB}$, Sigma, USA) was added into all solutions except for the nuclease solution.

\section{Histomorphological analyses}

After native enthesis tissue (NET), C-AEM, and O-AEM were fixed in $4 \%$ neutral buffered formalin and then washed with $0.9 \%$ saline to remove the residual formalin, they were decalcified with $10 \%$ EDTA and then embedded in paraffin. Serial $5 \mu \mathrm{m}$ thick sections from the midsagittal plane of each specimen were cut using a microtome a Leica RM 2125 Microtome (Reichert-Jung GmbH, Germany). After that, these sections were stained with hematoxylin and eosin (H\&E), 4',6-diamidino-2-phenylindole (DAPI), picrosirius red (PR), and toluidine blue (TB).

\section{DNA residue}

After the decellularization treatment, the samples ( $\mathrm{n}=6$ for each group) were frozen, and then freeze-dried using a lyophilizer at $-80{ }^{\circ} \mathrm{C}$ for $24 \mathrm{~h}$. The freeze-dried samples were weighed and then minced with tissue grinder. According to the DNeasy Blood \& Tissue protocol (Qiagen, Germany), $10 \mathrm{mg}$ of minced sample was digested with proteinase $\mathrm{K}$ (supplied with the kit) at $56{ }^{\circ} \mathrm{C}$ for $3 \mathrm{~h}$. The concentration of the purified DNA was quantified by PicoGreen DNA assay kit (Invitrogen, USA).

\section{Distribution and content of collagen and GAGs}

Paraffin-embedded NET, C-AEM, and O-AEM were sagittally sectioned into $5 \mu \mathrm{m}$ slices, then placed onto a $\mathrm{BaF} 2$ substrate. After being de-waxed and dried overnight, the sections were evaluated with synchrotron-radiation Fourier-transform infrared spectroscopy (SR-FTIR) (BL01B beamline of National Facility for Protein Science Shanghai and Shanghai Synchrotron Radiation Facility, China) to capture the infrared spectrum. The peak areas of carbohydrate $\left(1,140-985 \mathrm{~cm}^{-1}\right)$ and amide I $(1,720$ $\left.1,590 \mathrm{~cm}^{-1}\right)$ in the infrared spectrum were calculated to characterize the distribution and content of collagen and GAGs in the NET, C-AEM, and O-AEM.

\section{Scanning electron microscope (SEM)}

The samples ( $\mathrm{n}=6$ for each group) of the NET, C-AEM, and $\mathrm{O}-\mathrm{AEM}$ were fixed in $2.5 \%$ glutaraldehyde overnight at $4{ }^{\circ} \mathrm{C}$. After rinsing with PBS, these samples were immersed in $1 \% \mathrm{OsO}_{4}$ for $2 \mathrm{~h}$ and then dehydrated in a series of graded alcohols. Then, the samples were ready for drying in a critical point dryer with liquid CO2. After the dry samples were coated with gold, they were observed through SEM (Mira3 LMH, Tescan, Czech Republic), and their distribution and content of calcium (Ca) and phosphorus (P) were evaluated by energy dispersive spectrometry (EDS) analysis.

\section{Porosity}

The samples ( $\mathrm{n}=6$ for each group) of the NET, C-AEM, 
and $\mathrm{O}-\mathrm{AEM}$ were immersed into a $10-\mathrm{mL}$ volume cylinder with $5 \mathrm{~mL}$ of ethanol for $10 \mathrm{~min}$. Then, the volumes were measured (V1). After taking out the immersed scaffold, we measured the volume of the remaining ethanol (V2). Porosity was calculated according to the following equation: porosity $(\%)=(5-\mathrm{V} 2) /(\mathrm{V} 1-\mathrm{V} 2) \times 100 \%$.

\section{Water absorption}

In order to assess the property of AEM in holding body fluid in vivo, we measured the water absorption of the NET, $\mathrm{C}-\mathrm{AEM}$ and O-AEM. Briefly, the freeze-dried samples ( $n=6$ for each group) of the NET, C-AEM and O-AEM was weighed (M1), and then immersed into PBS for 24-h and weighed again (M2). Absorption was calculated according to the following equation: absorption $(\%)=(M 2-M 1) / M 1 \times$ $100 \%$.

\section{Tensile test}

Tensile properties of the NET, C-AEM, and O-AEM ( $n=8$ for each group) were measured as follows: after the NET, C-AEM, and O-AEM were lyophilized, the interface region of the NET, C-AEM, and O-AEM were trimmed into rectangle with cross-section area of $24 \mathrm{~mm}^{3}$. Then the bony and tendinous ends of the samples were embedded with resin. During the embedding process, care was taken to see that the resin material did not come in contact with the enthesis region of the NET, C-AEM, and O-AEM. Subsequently, the bony and tendinous ends of the samples were fixed into the grip of the mechanical testing system (MTS insight, MTS Systems Corp., USA) for failure testing. Each specimen was preloaded to $1 \mathrm{~N}$ and then loaded to failure at a rate of $30 \mathrm{~mm} / \mathrm{min}$. Failure load and stiffness were calculated from the load-displacement curve obtained from testing.

\section{GF residue}

The NET, C-AEM, and O-AEM (n=3 for each group) were carefully cut into bony, fibrocartilaginous, and tendinous scaffolds. To extract GFs from these scaffolds, they were suspended in a $1-\mathrm{mL}$ extraction buffer $(0.5 \mathrm{M}$ acetic acid, $50 \mathrm{mM}$ Tris- $\mathrm{HCl}, \mathrm{pH}=7.4,0.1 \times$ protease inhibitors), followed by stirring at $4{ }^{\circ} \mathrm{C}$ for 3 days. Then, the mixture was centrifuged at $12,000 \mathrm{~g}$ for $30 \mathrm{~min}$ at $4{ }^{\circ} \mathrm{C}$ to collect supernatant, which was then used to analyze the amount of bone morphogenetic protein-2 (BMP-2), transforming growth factor- $\beta 1$ (TGF- $\beta 1$ ), and connective tissue growth factor (CTGF) with ELISA kits (CUSABIO, China).

\section{Cell proliferation and viability}

After the C-AEM and O-AEM were sagittally sectioned into a $50 \mu \mathrm{m}$ slice using a freezing microtome (Leica CM1950; Nussloch, Germany), they were sterilized with ethylene epoxide in Xiangya hospital. Finally, $10^{3}$ human bone marrow mesenchymal stem cells (hBMSCs) were seeded onto the C-AEM, O-AEM, and tissue culture polystyrenes (TCPS) as control. After 1-, 3-, 7-, 10-, and 14-day cultures, cell proliferation on the C-AEM, O-AEM, and TCPS were quantified with CCK8 kits (70-CCK8100, MultiSciences, China). Additionally, $5.0 \times 10^{4} \mathrm{~h}$ BMSCs were seeded onto the C-AEM, O-AEM, and TCPS as control. After a 7-day culture, cell viability was evaluated with a live/dead assay kit (40747ES76, Yeasen, Shanghai, China). The green- and red-stained cells on the C-AEM, O-AEM, and TCPS were captured by fluorescence microscopy with excitation wavelengths of $488 / 594 \mathrm{~nm}$ to quantify cell viability.

\section{Inflammatory responses}

RAW 264.7 cells (ATCC) were co-cultured with the C-AEM and O-AEM using 24-well transwell inserts (Costar 3422, Corning, USA) to evaluate their pro-inflammatory property. In the lower chamber, $5.0 \times 10^{4}$ cells/wells of RAW 264.7 cells were cultured in a complete medium (highglucose DMEM + 10\% FBS + 1\% antibiotics, Gibco, USA). According to the different media in the upper compartment, the experiment was divided into TCPS group (only complete medium), lipopolysaccharide (LPS) group (complete medium with $10 \mu \mathrm{g} / \mathrm{mL}$ LPS), C-AEM group (complete medium with $\mathrm{C}-\mathrm{AEM}$ ), and O-AEM group (complete medium with O-AEM). After a 3-day culture, the morphology of RAW 264.7 cells was captured, and TNF- $\alpha$, IL-6, and IL-1 $\beta$ in the supernatant were measured with ELISA kits (Multi Sciences, China).

\section{bBMSC differentiation on AEM}

A total of $5 \times 10^{4} \mathrm{hBMSC}$ were seeded on the section of the C-AEM, O-AEM, and TCPS. An immunofluorescence assay was used to observe the expression of Runx-2, Sox-9, and $S c x$ in the hBMSCs after a 14-day culture using antiRunx-2 antibody (Abcam), anti-Sox-9 antibody (Abcam), 
and anti-Scx antibody (Abcam). Anti-collagen II antibody (Abcam) was used to label collagen II for defining the distinct regions of the scaffold. In addition, quantitative real time-polymerase chain reaction (qRT-PCR) was used for quantitatively evaluating the expression of osteogenic (Runx-2, Ocn), chondrogenic (Sox-9, Acan), and tenogenic $(S c x, T n m d)$ genes in hBMSCs. The housekeeping gene $(G A P D H)$ was used for normalization. The results are shown as fold change related to the hBMSCs in the TCPS group. The primer sequences for these genes are listed in Table S1.

\section{Experimental animals}

The adult male beagle dogs aged 8 months and weighing $9.5-10.5 \mathrm{~kg}$ were used in this study. The dogs were individually housed in an unrestricted cage and fed ad libitum. These healthy dogs were allowed to accommodate to the environment for 1 week before experiment. All procedures complied with the Animals (Scientific Procedures) Act 1986 and this study was approved by the Animal Ethics Committee of Xiangya Hospital, Central South University (No. 2019030517).

\section{Surgical methods}

In this study, dogs were anesthetized with intravenous pentobarbital sodium $(10 \mathrm{mg} / \mathrm{kg})$, intubated, and maintained on isoflurane in oxygen (3\%). The IT was approached and detached from its insertion at the greater tuberosity. The residual fibrocartilage at the insertion was decorticated with a grinding wheel saw. In the CTL group, two bony tunnels were made at the greater tuberosity with a 1-mm-diameter drill bit, and two medial anchors were inserted into the upper and lower poles of footprints with a distance of $4 \mathrm{~mm}$. Then, the ruptured tendon end was repaired and put back to its insertion using two transosseous modified Mason-Allen sutures (AR-1324BF, Arthrex, Naples, Germany). In the C-AEM or O-AEMaugmented groups, two anchors were inserted into the same position of the CTL group. Two suture limbs from one anchor were passed through the pre-drilled tunnels on the bony region (BR) of the AEM, and then knotted them together to fix the bony end of the AEM to the IT footprint. Then, one limb from each anchor was also passed in parallel through the bone cortex of the lateral margin of footprints and knotted with another limb to make the bony part of the AEM firmly fixed to the footprint area. After the IT end was horizontally split into two layers, one suture limb was passed through the tendon end with Kessler repair to pull the tendon end to the footprint area and avoid excessive tension on the AEM at an early phase. Then, the tendinous part of the AEM was sandwiched by the two-layer tendon ends and the edges of this sandwich construct were slightly sutured together with continuous 5-0 PDS sutures (Ethicon). The wounds were irrigated with normal saline and closed in layers. Postoperatively, the dogs were allowed to freely engage in cage activity with a cast immobilization to protect the operative limb further from weight bearing and separation of the junction reattachment. A 7-day painkiller (Tramadol; Grunenthal $\mathrm{GmbH}$ ) was given after the operation (P.O, $\mathrm{q} 8 \mathrm{~h}, 50 \mathrm{mg}$ ). Amoxicillin and clavulanate potassium tablets (Zhuhai united laboratories Co., Ltd., China) were given as a prophylactic antibiotic for 5 days (P.O, q12h, $475 \mathrm{mg}$ ). At postoperative week 8 , ten dogs were sacrificed, and the IT and attached bony block were collected.

\section{Histological assessment}

After the harvested specimens were fixed in $4 \%$ paraformaldehyde and decalcified with $10 \%$ EDTA, they were embedded in paraffin and sagittally cut into $5 \mu \mathrm{m}$ sections along the long axis of the IT. These sections were then stained with $\mathrm{H} \& \mathrm{E}$, safranin $\mathrm{O} /$ fast green ( $\mathrm{SO} /$ FG) and masson trichrome (MT), and observed under a light or polarized microscope (Eclipse CI, Nikon, Japan). The healing site was semi-quantitatively analyzed by two blinded observers (QS and YC) using a histological score system adapted from Ide et al. and Shah et al. (Table S2) $(32,33)$. The amount of fibrocartilage formation at the healing site was evaluated by quantifying the area of metachromasia using Image-Pro Plus (version 6.0.0; Media Cybernetics Inc.).

\section{Statistical analysis}

Statistical analyses were performed with the SPSS 25.0 software (IBM, USA). All quantitative data were shown as mean \pm standard error of mean, and differences between the two groups were evaluated using Student's $t$-test. Differences above two groups were evaluated using oneway analysis of variance (ANOVA) with post hoc test. The histological scores among the CTL, C-AEM, and O-AEM were calculated using Kruskal-Wallis testing. Statistical significance was set at $\mathrm{P}<0.05$. 


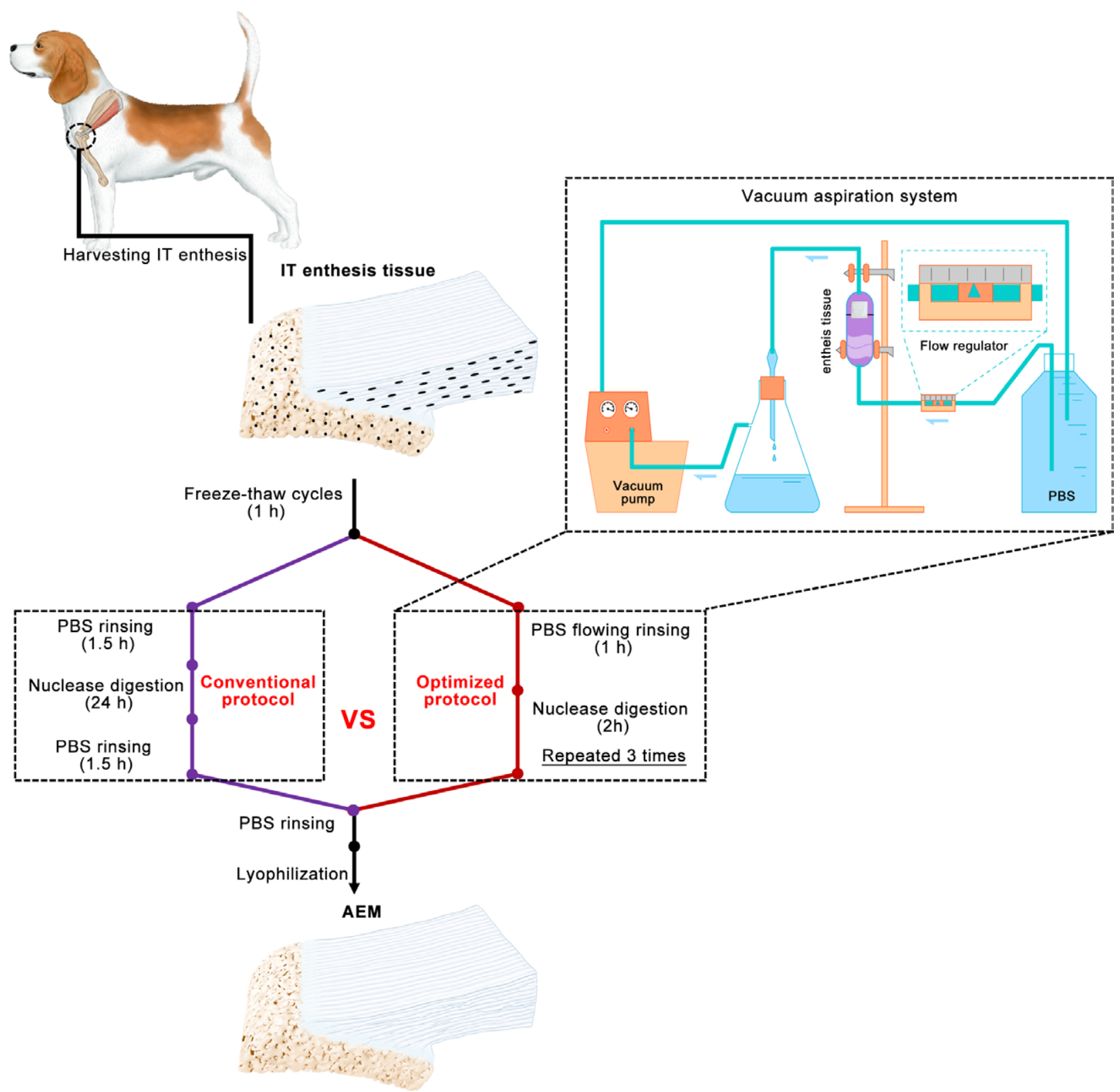

Figure 1 Overview of the conventional and optimized acellular protocols. Schematic representation of custom-designed VAS for large-sized enthesis decellularization. VAS, vacuum aspiration system; IT, infraspinatus tendon; PBS, phosphate buffer saline; AEM, acellular enthesis matrix.

\section{Results}

\section{Time for decellularization with conventional and optimized protocols}

In our optimized protocol, we shortened the time for decellularization from 28 to $10 \mathrm{~h}$ (Figure 1). The protocol was modified according to Liu et al. from a conventional protocol that includes freeze-thaw cycles $(1 \mathrm{~h})$, PBS rinsing (1.5 h), nuclease digestion (24 h), and PBS rinsing $(1.5 \mathrm{~h})(34)$. The optimized protocol includes freeze-thaw cycles $(1 \mathrm{~h})$, PBS rinsing $(1 \mathrm{~h})$, and nuclease digestion ( $2 \mathrm{~h}$, repeated three times). This VAS provided a negative pressure environment and flowed PBS rinsing on the enthesis tissue, thus quickly removing cellular components in enthesis while properly preserving its original matrix characteristics. 


\section{Physicochemical characteristics of AEM}

\section{Morphology}

Gross photographs of the AEM are shown in Figure $2 \mathrm{~A}$. Their sizes were approximately $3.0 \mathrm{~cm} \times 0.8 \mathrm{~cm} \times 0.5 \mathrm{~cm}$. $\mathrm{H} \& \mathrm{E}, \mathrm{DAPI}, \mathrm{PR}$, and TB staining were used together to evaluate the acellular effect and the preservation of major components of enthesis ECM (collagen and GAGs), as shown in Figure 2B. H\&E staining showed that the cellular components of the C-AEM and O-AEM were totally removed, and the histomorphology of the native enthesis was properly preserved. DAPI-positive cell nuclei were rarely present in the two AEM groups. In addition, PR staining slices, which were observed under polarized light, confirmed the preservation of collagen in the C-AEM and $\mathrm{O}$-AEM. Furthermore, TB staining was positive in the fibrocartilaginous region (FR) of the C-AEM and O-AEM, while the $\mathrm{O}-\mathrm{AEM}$ presented a slightly higher blue intensity than the C-AEM.

\section{Distribution and content of major ECM components at the AEM by SR-FTIR}

In this study, we applied SR-FTIR to characterize the distributions of collagen and GAG content comparatively among the NET, C-AEM, and O-AEM. As showed in Figure 2, the collagen distributions in the C-AEM and O-AEM were similar, but the collagen content in the BR of the O-AEM lost approximately $13.50 \%$ of the collagen content with respect to the BR of NET $(\mathrm{P}<0.05)$, while the $\mathrm{C}$-AEM lost approximately $24.49 \%$ in the $\mathrm{BR}(\mathrm{P}<0.05)$. Interestingly, the fibrocartilaginous and tendinous regions (TRs) of the NET, C-AEM, and $\mathrm{O}-\mathrm{AEM}$ showed similar values in collagen content without significant difference $(\mathrm{P}>0.05)$. Concerning GAGs, their distribution in the C-AEM and O-AEM were partly reserved after decellularization, but the content in the fibrocartilaginous and TRs of the O-AEM was preserved much better than in the C-AEM $(\mathrm{P}<0.05)$. These results indicate that the optimized protocol can effectively decellularize the cellular component of the NET while preserving the ECM components much better than the conventional approach.

\section{DNA residence}

The content of DNA was $0.029 \pm 0.010 \mu \mathrm{g} / \mathrm{mg}$ (dry weight) in the C-AEM and $0.027 \pm 0.012 \mu \mathrm{g} / \mathrm{mg}$ in the O-AEM, which was significantly lower than the content in the NET $(1.035 \pm 0.093)(\mathrm{P}<0.001)$ (Figure 2C).

\section{Microstructure}

According to the histological (Figure 2) and SEM images (Figure 3A), AEM prepared with either the conventional (C-AEM) or optimized (O-AEM) protocol preserved its native structure well, and no crumpled cell debris was visualized. The porosity was quantitatively measured: $25.26 \% \pm 1.71 \%$ for the NET, $32.70 \% \pm 3.27 \%$ for the $\mathrm{C}-\mathrm{AEM}$, and $31.90 \% \pm 2.47 \%$ for the O-AEM $(\mathrm{P}<0.01)$, as shown in Figure 3B. Water absorption was approximately $133.50 \% \pm 31.35 \%$ for the C-AEM, $139.76 \% \pm 31.83 \%$ for the O-AEM, and $91.54 \% \pm 10.15 \%$ for the NET $(\mathrm{P}<0.05)$, as shown in Figure $3 B$.

\section{Mineral preservation}

EDS analysis showed a similar distribution and content of $\mathrm{Ca}$ and $\mathrm{P}$ in the NET, C-AEM, and O-AEM (Figure $3 A$ ). Quantitative analysis confirmed that the calcium content in the bony and calcified regions of the C-AEM and O-AEM presented a slight decrease compared to the bony and calcified region of the NET without any significant difference. The calcium content in the uncalcified and TRs of the NET, C-AEM, and O-AEM was very low, with no significant difference (Figure 3C). P content showed a similar distribution to that of the calcium content (Figure 3C).

\section{GFs at the AEM}

To evaluate the influence of decellularization on the reservation of GFs in the AEM, BMP-2, TGF- $\beta 1$, and CTGF were analyzed in extracts from the BR, FR, and TRs of the NET, C-AEM, and O-AEM by ELISA (Figure 4). The BMP-2 in the BRs of the NET, C-AEM, and O-AEM showed significantly higher content than in other regions, but no significant difference was found among these BRs. Interestingly, ELISA analysis confirmed that the TGF- $\beta 1$ was preserved better in the FR of scaffolds than in other regions. CTGF showed a significantly higher content in the TR of scaffolds without significant difference among the TRs of the NET, C-AEM, and O-AEM.

\section{Tensile properties}

After lyophilization, the tensile properties of the NET, $\mathrm{C}-\mathrm{AEM}$, and O-AEM were measured by a mechanical testing machine (Figure $5 A$ ). During the test, all the samples ruptured at the middle part of the tendon. Failure load $(\mathrm{N})$ and stiffness $(\mathrm{N} / \mathrm{mm})$ were measured from the recorded load-displacement. The O-AEM and NET performed similarly in failure load $(\mathrm{N})$, which was significantly higher than for the C-AEM (Figure 5B). Concerning stiffness 
A
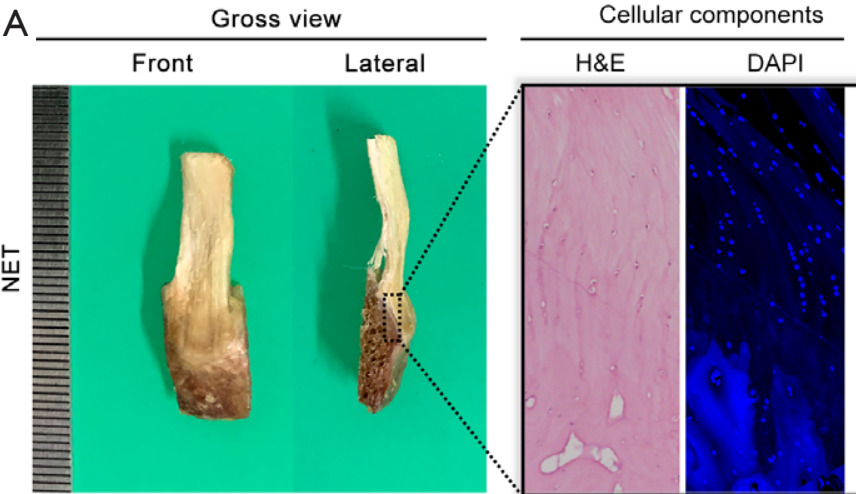

\begin{tabular}{|c|c|c|c|}
\hline \multicolumn{2}{|c|}{ Collagens } & \multicolumn{2}{|c|}{ GAGs } \\
\hline PR & SR-FTIR & TB & SR-FTIR \\
\hline
\end{tabular}
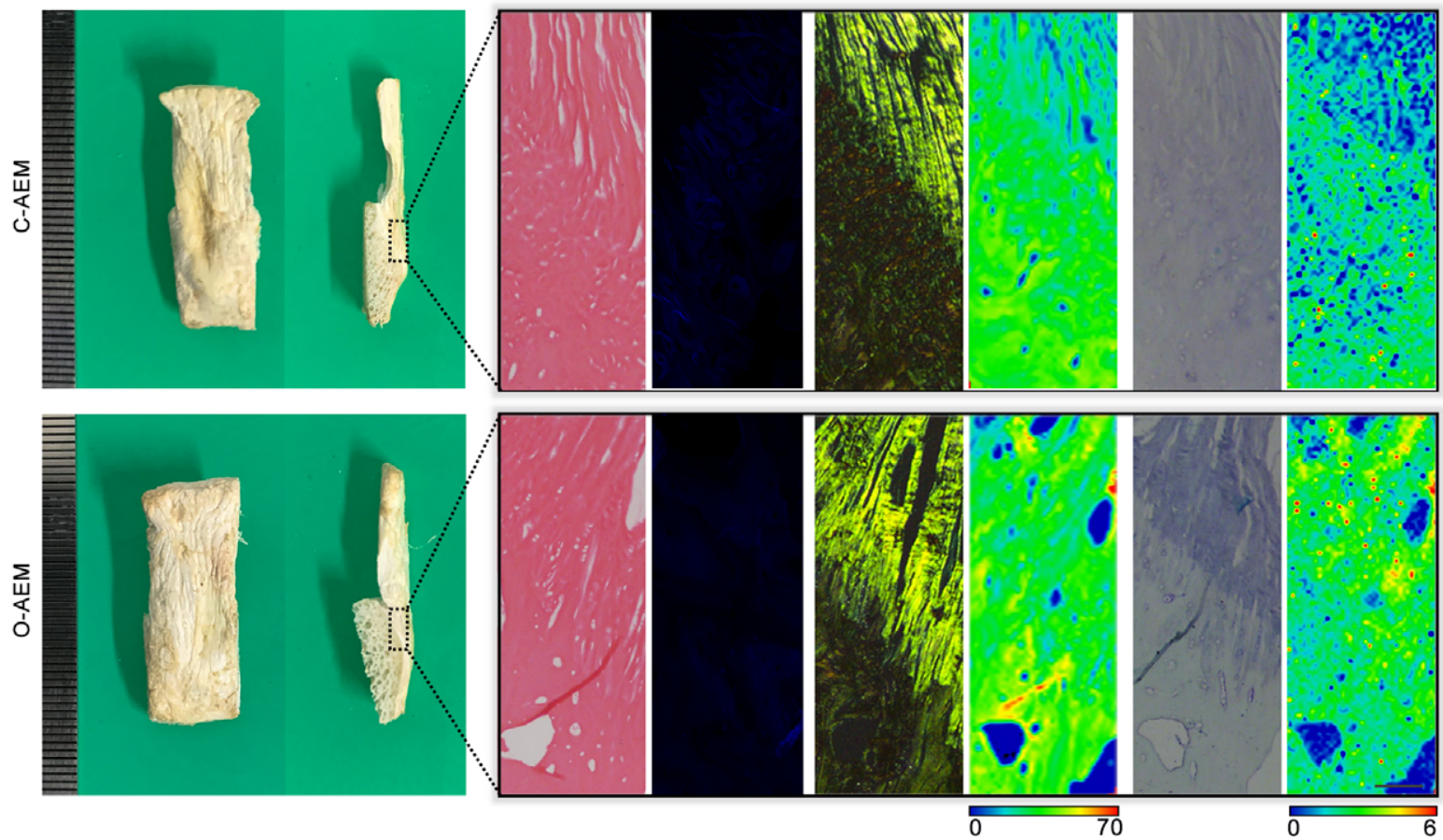

B

- NET * C-AEM \& O-AEM
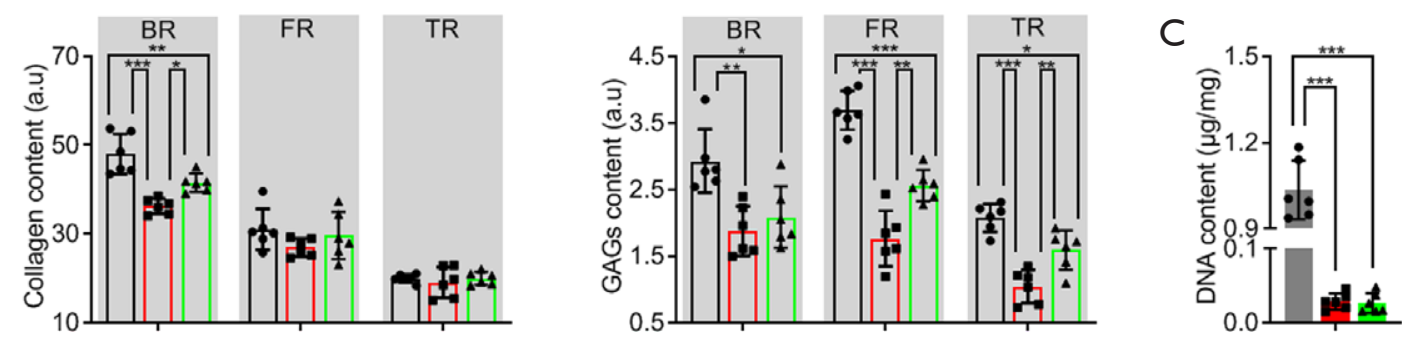

Figure 2 Morphological characteristics of the AEM fabricated with conventional or optimized protocol. (A) Gross features, cellular components, collagens distribution and content, GAGs distribution and content of the NET, C-AEM or O-AEM. H\&E and DAPI for observing cellular components. PR together SR-FTIR for evaluating the distribution and content of collagen. TB combined with SR-FTIR for evaluating the distribution and content of GAGs. Bar $=100 \mu \mathrm{m}$; (B) collagen and GAGs contents in the BR, FR or TR of the NET, C-AEM or O-AEM ( $\mathrm{n}=6)$; (C) DNA content in the NET and the two kinds of AEM $(\mathrm{n}=5) .{ }^{*}, \mathrm{P}<0.05 ;{ }^{* *}, \mathrm{P}<0.01$; ***, $\mathrm{P}<0.001$. AEM, acellular enthesis matrix; GAG, glycosaminoglycan; NET, native enthesis tissue; H\&E, hematoxylin and eosi; DAPI, 4',6-diamidino-2-phenylindole; PR, picrosirius red; SR-FTIR, synchrotron-radiation Fourier-transform infrared spectroscopy; TB, toluidine blue; BR, bony region; FR, fibrocartilaginous region; TR, tendinous region. 
A
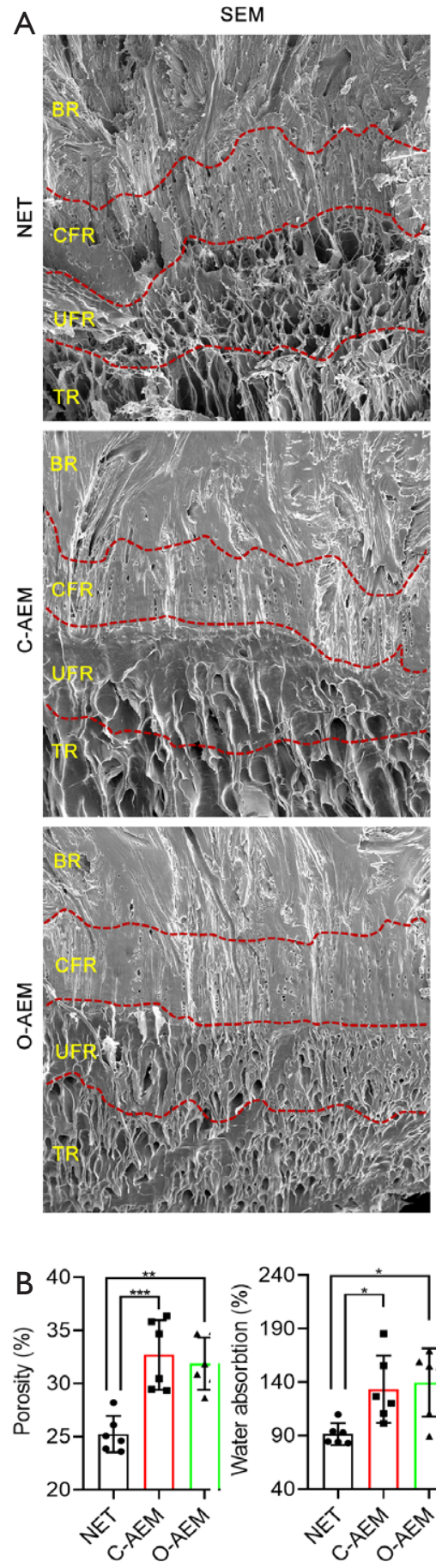

Ca distribution
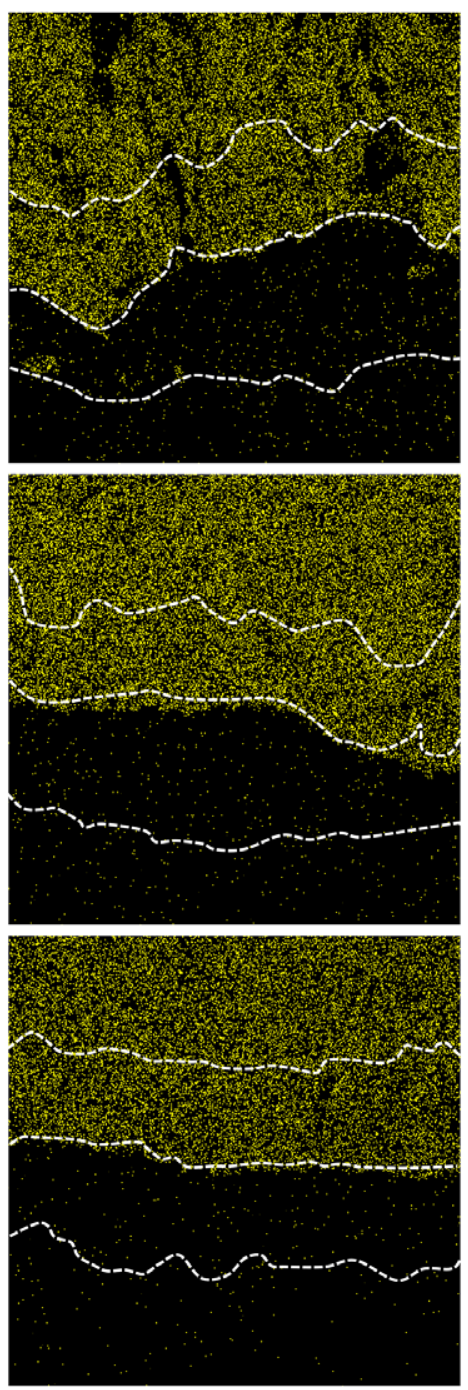

$\square$ NET
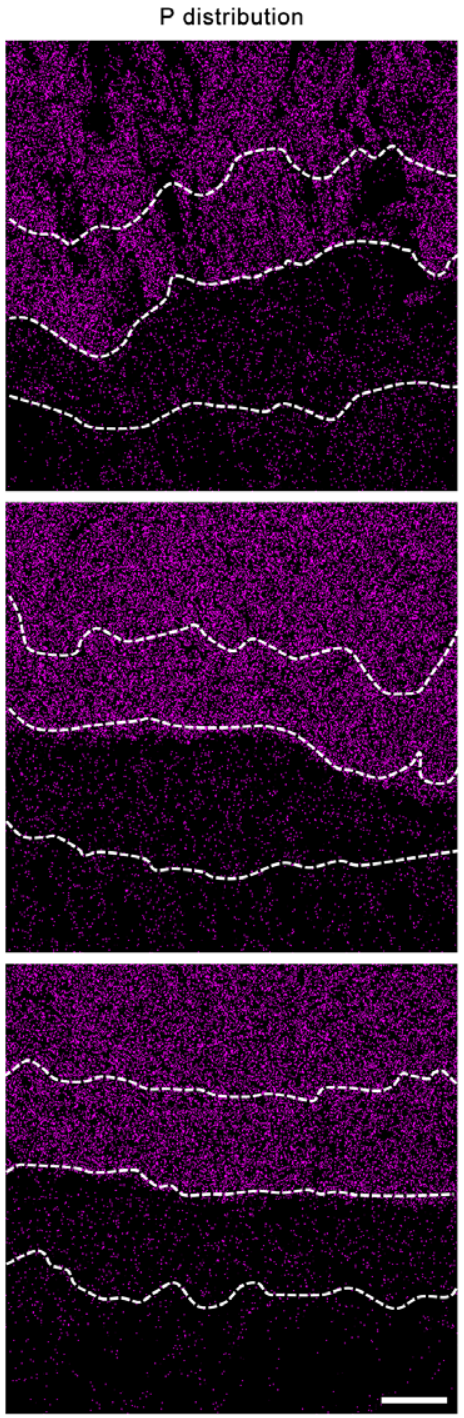

$\square$ C-AEM $\square$ O-AEM

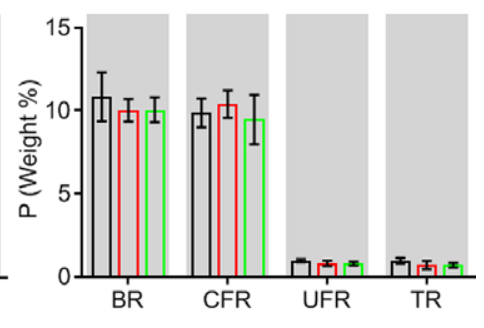

Figure 3 Mineral preservation of the NET, C-AEM, or O-AEM. (A) SEM images, EDS mappings of Ca and P in the NET, C-AEM or O-AEM. Bar $=200 \mu \mathrm{m}$; (B) comparison of the porosity and water absorption for the NET, C-AEM or O-AEM (n=6); (C) Ca and P content in the NET, C-AEM or O-AEM (n=6). * $\mathrm{P}<0.05 ;{ }^{* *}, \mathrm{P}<0.01$; ${ }^{* *}, \mathrm{P}<0.001$. SEM, scanning electron microscope; EDS, energy dispersive spectrometry; Ca, calcium; P, phosphorus; NET, native enthesis tissue; AEM, acellular enthesis matrix; BR, bony region; CFR, calcified fibrocartilaginous region; UFR, uncalcified fibrocartilaginous region; TR, tendinous region. 

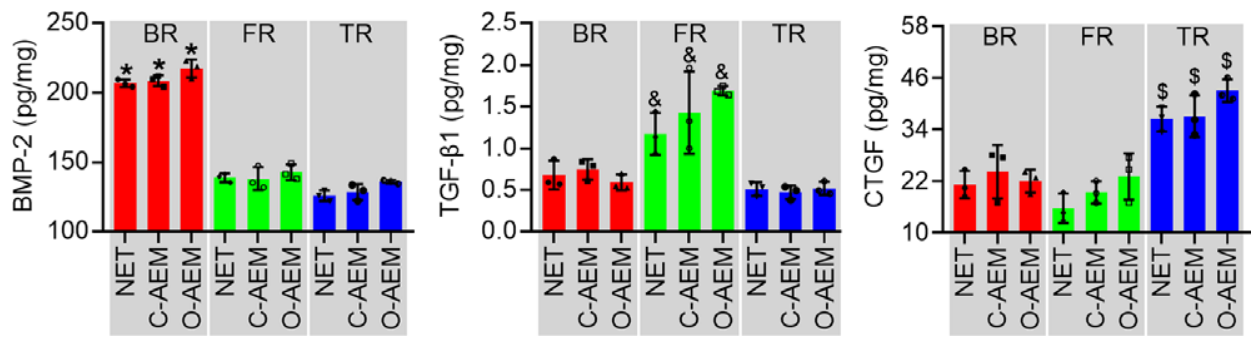

Figure 4 Conventional or optimized acellular protocol on the preservation of GFs (BMP-2, TGF- $\beta 1$ and CTGF) in lyophilized NET, C-AEM or O-AEM ( $\mathrm{n}=3) .{ }^{*}, \mathrm{P}<0.05$ compared with the FR and TR of scaffolds; ${ }^{*}, \mathrm{P}<0.05$ compared with the BR and TR of scaffolds; ${ }^{\text {, }}$, $\mathrm{P}<0.05$ the bony and FR of scaffolds. GF, growth factor; BMP-2, bone morphogenetic protein- 2 ; TGF- $\beta 1$, transforming growth factor- $\beta 1$; CTGF, connective tissue growth factor; NET, native enthesis tissue; AEM, acellular enthesis matrix; FR, fibrocartilaginous region; TR, tendinous region; BR, bony region.

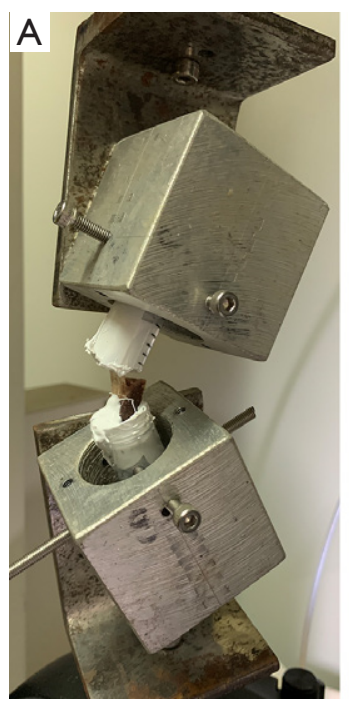

- NET - C-AEM \& O-AEM
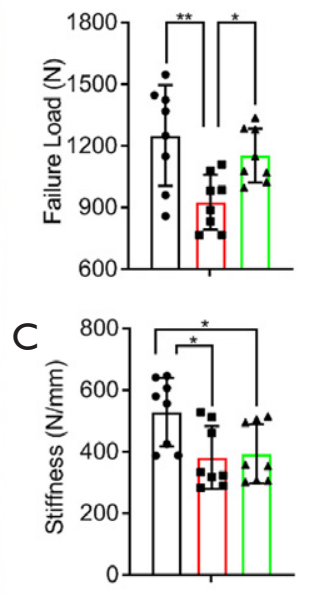

Figure 5 Tensile properties of the NET, C-AEM or O-AEM. (A) Tensile testing of the specimen using a custom-made jig that includes an upper clamp and a lower clamp to the resin embedded IT and bony block in humerus, respectively; $(\mathrm{B}, \mathrm{C})$ the failure load and stiffness of the NET, C-AEM or O-AEM ( $=8)$. *, $\mathrm{P}<0.05$; **, $\mathrm{P}<0.01$. NET, native enthesis tissue; AEM, acellular enthesis matrix; IT, infraspinatus tendon.

$(\mathrm{N} / \mathrm{mm})$, the values in both C-AEM and O-AEM were significantly lower than the NET, but the O-AEM showed a slightly higher value than the C-AEM without significant difference (Figure 5C).

\section{Biological characteristics of AEM}

\section{Cells proliferation and viability}

To evaluate the effects of C-AEM and O-AEM on BMSCs proliferation, a cell counting kit- 8 was used to examine hBMSCs proliferation cultured on the C-AEM and O-AEM. This assay showed that hBMSCs seeded on the C-AEM and O-AEM proliferated similarly to TCPS as negative control (Figure 6A). On day 3 after seeding, the live/dead assay showed that most hBMSCs were stained fluorescent green (living cells) and very few red (dead cells). The quantitative analysis showed that hBMSCs viability on the C-AEM and O-AEM was lower than on the TCPS without any significant difference (Figure 6B).

\section{Inflammatory activities}

Pro-inflammatory cytokines (TNF- $\alpha$, IL-6 and IL-1 $\beta$ ) were measured to evaluate the inflammatory responses of macrophages (RAW 264.7) for the C-AEM and O-AEM. As shown in Figure 6C, the morphology of RAW 264.7 cultured on TCPS, C-AEM, and O-AEM was similar, without evident cell tentacles, while most of RAW 264.7 under LPS (as positive control) clearly showed cell tentacles. Additionally, RAW 264.7 cultured on C-AEM and O-AEM generated a slightly higher level of TNF- $\alpha$, IL- 6 , and IL- $1 \beta$ than those cultured on TCPS at day 3 ( $P>0.05$ for all), but still significantly lower than the LPS-treated group (Figure 6D).

\section{hBMSC differentiation on AEM}

Osteogenic, chondrogenic, and tenogenic differentiation of hBMSCs on the BR, FR, and TRs of the C-AEM, $\mathrm{O}-\mathrm{AEM}$, and TCPS were assessed by qRT-PCR and cellular immunofluorescence. As shown in Figure $7 A$, the mRNA levels of osteogenic (Runx-2, Ocn), chondrogenic (Sox-9, Acan), and tenogenic (Scx, Tnmd) marker genes showed varying stem cell differentiation as a result of the influence of the TCPS, C-AEM, and O-AEM on day 14 after hBMSCs seeding. The expression level of osteogenic 


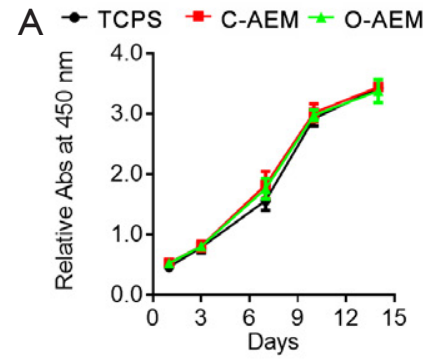

C

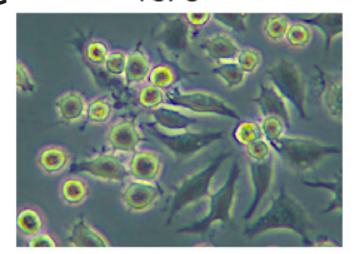

D

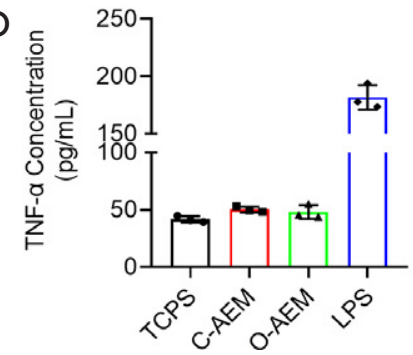

B TCPS

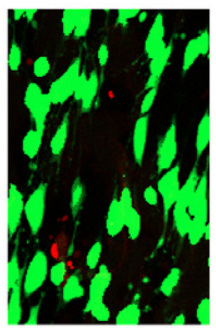

C-AEM
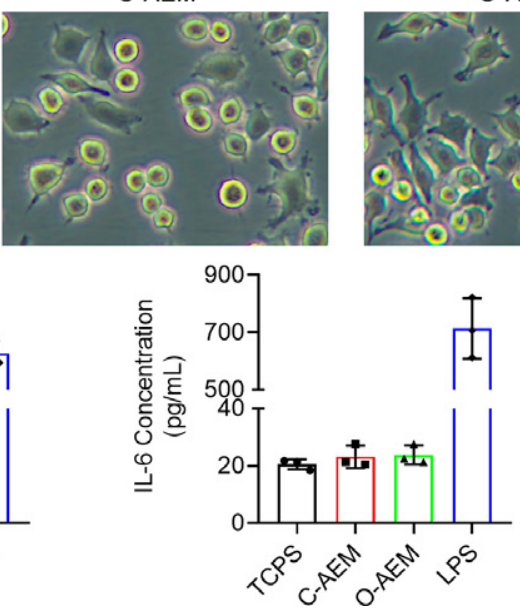

C-AEM

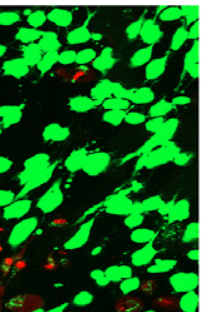

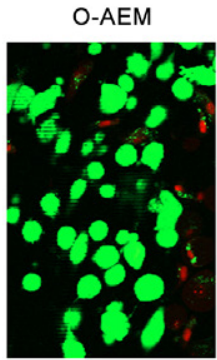

O-AEM

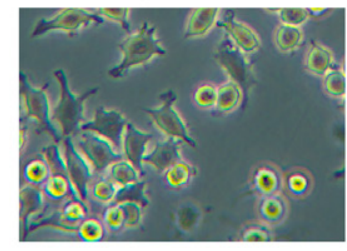

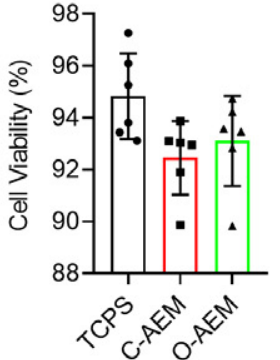

LPS
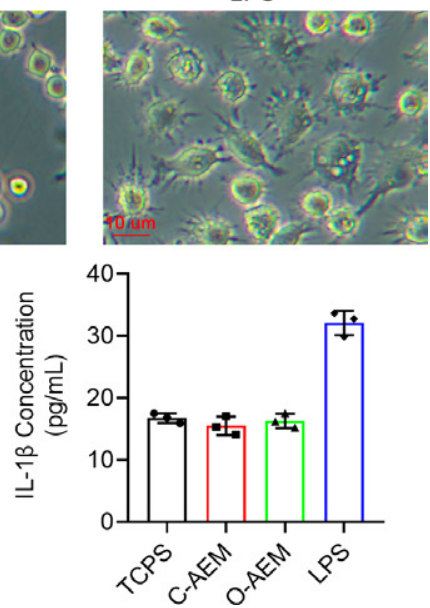

Figure 6 Cells proliferation and viability and inflammatory activities of the NET, C-AEM or O-AEM. (A) Comparative cell proliferation assay of hBMSCs seeded on the TCPS, C-AEM and O-AEM ( $\mathrm{n=3}$ ); (B) live/dead cell analysis for the TCPS, C-AEM and O-AEM on which hBMSCs seeded for 3 days. Representative images show the live (green) and dead (red) hBMSCs in the TCPS, C-AEM and O-AEM, and the viability analysis for the cells on the TCPS, C-AEM and O-AEM (n=6). Bar =100 $\mu \mathrm{m}$; (C) representative RAW 264.7 morphology after 3 days of stimulation by TCPS, C-AEM, O-AEM or LPS. Bar $=10$ or $100 \mu \mathrm{m}$; (D) pro-inflammatory cytokine (TNF- $\alpha$, IL-6, and IL-1 $\beta$ ) release by stimulation with TCPS, C-AEM, O-AEM or LPS detected by ELISA assay ( $\mathrm{n}=3)$. hBMSC, human bone marrow mesenchymal stem cell; TCPS, tissue culture polystyrenes; AEM, acellular enthesis matrix; LPS, lipopolysaccharide.

genes in the BR of the O-AEM was significantly higher than in the C-AEM and TCPS $(\mathrm{P}<0.05)$. Similarly, the chondrogenic genes only expressed significantly in the FR of the O-AEM. Concerning the tenogenic gene expression, the TR of the O-AEM showed the largest value among the TCPS, C-AEM, and O-AEM. Our data suggest that the O-AEM showed better region-specific differentiation inducibility for in vitro hBMSCs. To assess this aspect further, we evaluated the expression of Runx-2, Sox-9, and Scx in hBMSCs after culturing on the TCPS, C-AEM, and O-AEM for 14 days through acellular immunofluorescence assay (Figure $7 B$ ). It is clear that hBMSCs in the BR, FR, and TRs of the O-AEM expressed the Runx-2, Sox-9, and Scx proteins, as opposed to the TCPS and C-AEM.

\section{Histological evaluation on enthesis regeneration}

No evidence of infection, rejection, or untoward immune reactions was detected for any repairs based on histological assessments. Using a canine IT repair model (Figure $8 A$ ), a great deal of loose and disorganized Sharpey fibers were observed at the insertion in the CTL group (Figure $8 B$ ). Additionally, there was a limited formation of fibrocartilaginous tissue at the healing interface. The $\mathrm{C}-\mathrm{AEM}$ and O-AEM groups showed better enthesis regeneration than the CTL group, but the regenerated enthesis in the O-AEM group was better in terms of histological features than that of the C-AEM group (Figure 8B). Histologically, the tendons in the C-AEM and O-AEM groups showed denser well-orientated collagen and more spindle-shaped nuclei than the CTL. Highly fibrocartilaginous tissue was observed in the O-AEM group, which is characteristic of mature and well-orientated chondrocytes and a matrix full of collagens and GAGs. In statistical terms (Figure 8C), using a modified histological 

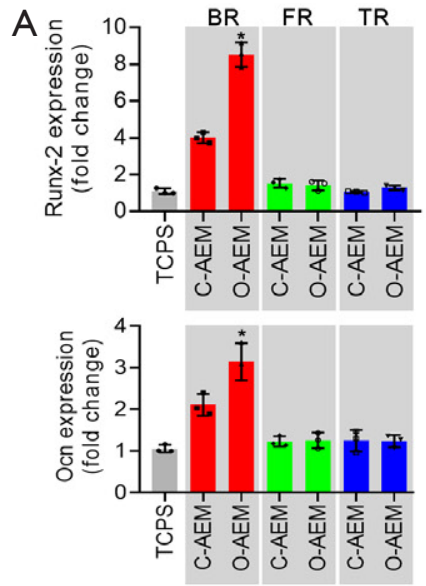

B
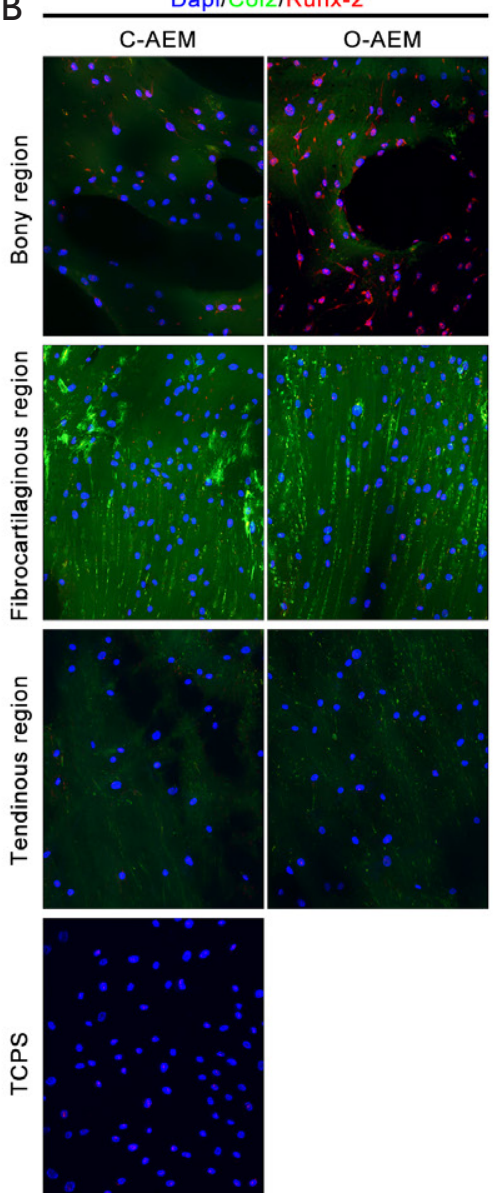
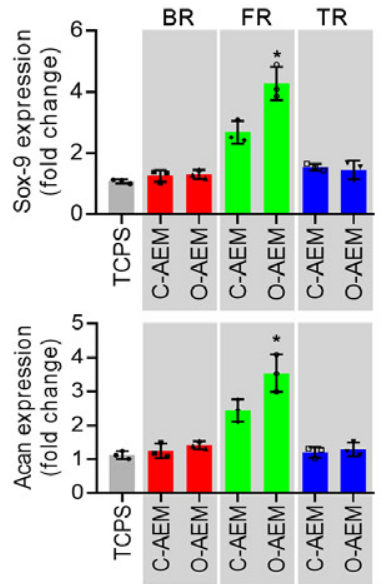

Dapi/Col2/Sox-9
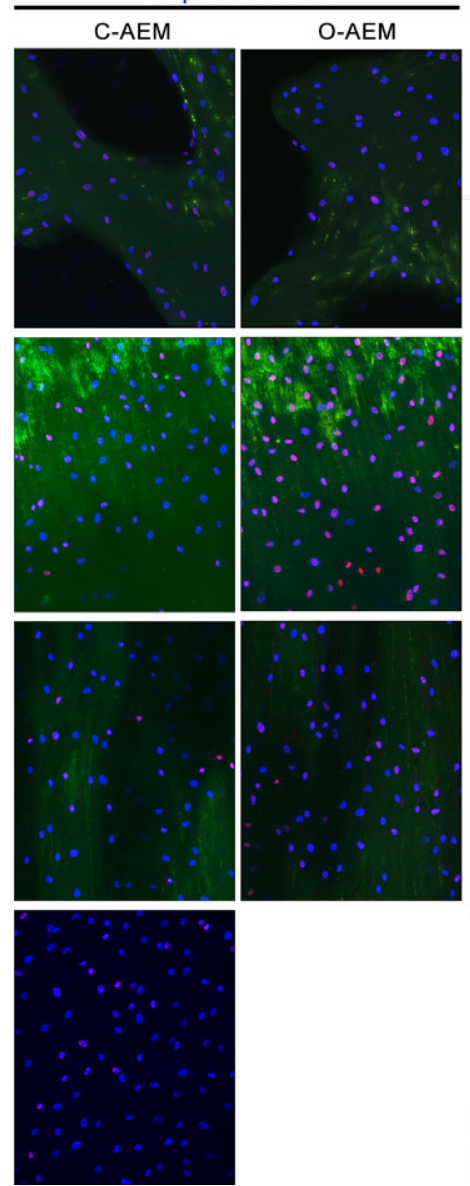
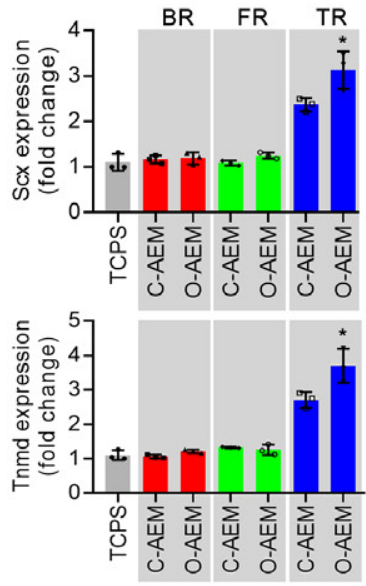

Dapi/Col2/Scx

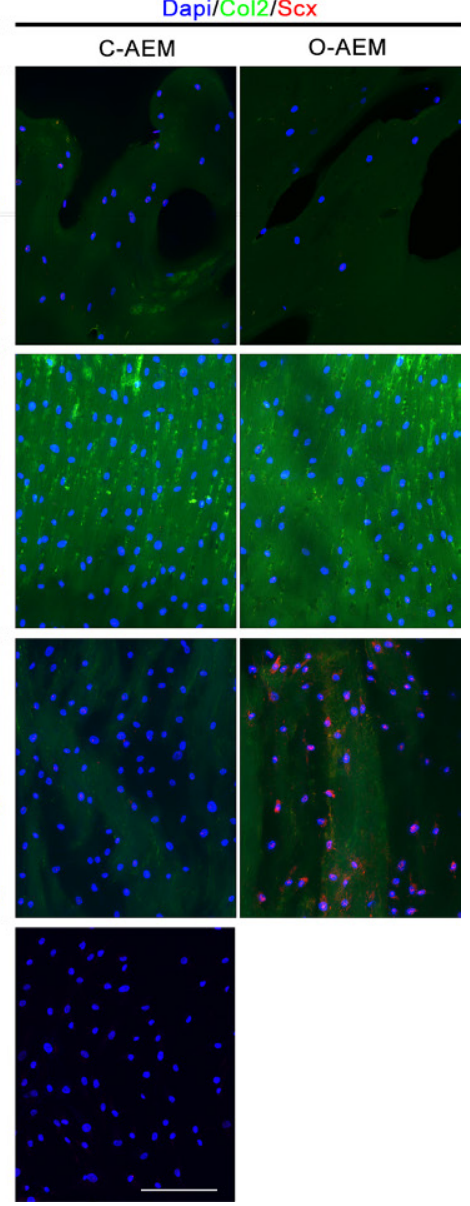

Figure 7 Osteogenic, chondrogenic, and tenogenic differentiation of hBMSCs in the C-AEM, O-AEM, and TCPS were assessed by qRTPCR and cellular immunofluorescence. (A) qRT-PCR analysis shows that the expression of osteogenic (Runx-2 and Ocn), chondrogenic (Sox-9 and Acan), and tenogenic ( $S c x$ and Tnmd) genes in hBMSCs cocultured on the TCPS or the BR, FR, TR of C-AEM and O-AEM for 14-day $(\mathrm{n}=3)$; (B) cellular immunofluorescence analysis of Runx-2, Sox-9 and Scx expression on hBMSCs cultured with TCPS or the BR, FR, TR of C-AEM and O-AEM for 14-day. Bar $=100 \mu \mathrm{m}$. *, P<0.05 compared among TCPS and the BR, FR, TR of C-AEM and O-AEM. qRT-PCR, quantitative real time-polymerase chain reaction; hBMSC, human bone marrow mesenchymal stem cell; TCPS, tissue culture polystyrenes; $\mathrm{BR}$, bony region; FR, fibrocartilaginous region; TR, tendinous region; AEM, acellular enthesis matrix. 
A

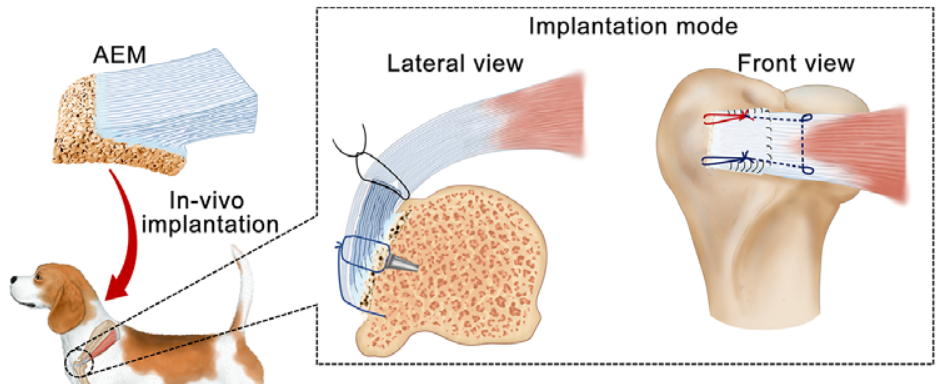

B
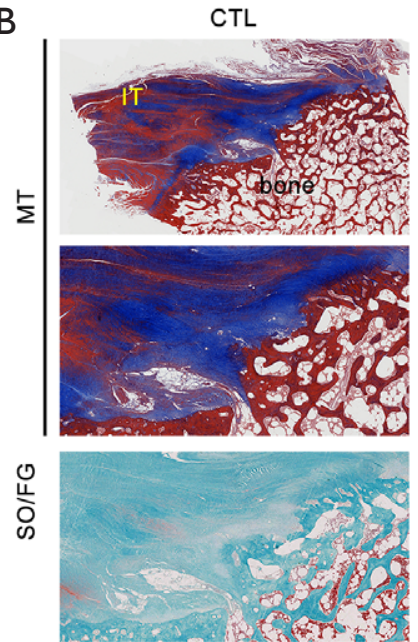

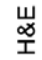

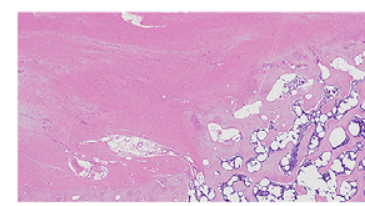

C-AEM
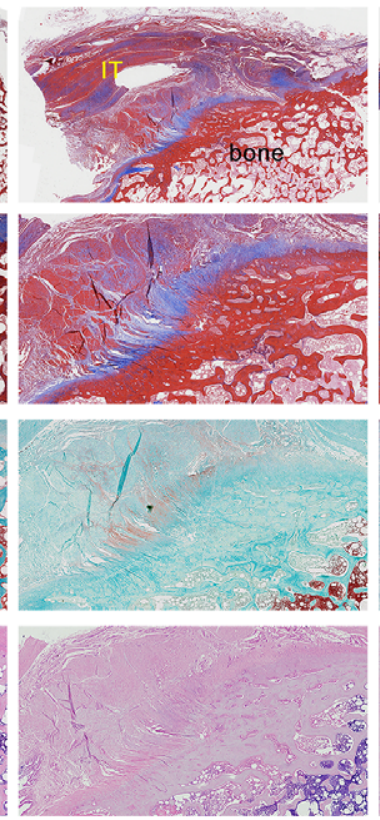

O-AEM
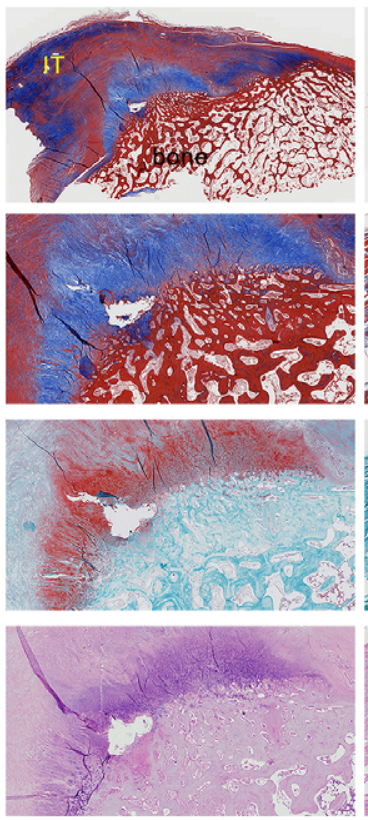

Native IT enthesis
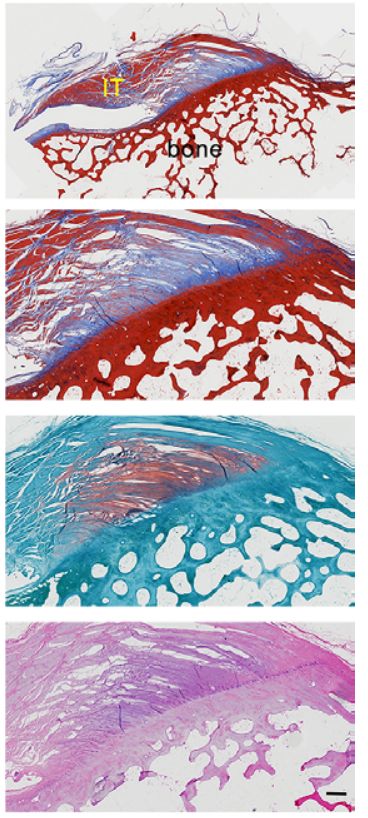
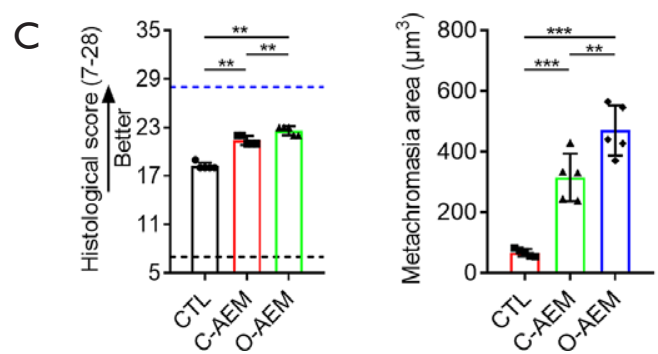

Figure 8 Histological evaluation on enthesis regeneration. (A) Canine IT repaired with AEM; (B) regenerated IT enthesis sections stained with H\&E, SO/FG, and MT at postoperative week 12. Healthy IT enthesis was used as a native control (NET). Bar =500 $\mu$ m; $(\mathrm{C})$ histological scores for the regenerated IT enthesis, and metachromasia area at the healing site. Blue and black dotted lines, respectively, indicate the perfect score (28 points) and worst score (7 points) in the histological scoring system. Bar $=200 \mu \mathrm{m} . \mathrm{n}=5$. ${ }^{* *}, \mathrm{P}<0.01 ;{ }^{* * *}$, $\mathrm{P}<0.001$. IT, infraspinatus tendon; AEM, acellular enthesis matrix; H\&E, hematoxylin and eosi; SO/FG, safranin O/fast green; MT, masson trichrome; NET, native enthesis tissue. 
scoring system, the histological scores for the regenerated enthesis in the $\mathrm{O}-\mathrm{AEM}$ group were higher than those of the $\mathrm{C}-\mathrm{AEM}$ and CTL group $(\mathrm{P}<0.05)$. The metachromasia area at the healing site was significantly increased in both C-AEM and O-AEM groups compared with the CTL group $(\mathrm{P}<0.05)$, and significant difference was found between the $\mathrm{C}-\mathrm{AEM}$ and $\mathrm{O}-\mathrm{AEM}$ groups $(\mathrm{P}<0.05)$.

\section{Discussion}

Biological scaffolds derived from acellular ECM have been increasingly used in the field of tissue-engineering. Various acellular protocols were investigated for preparing acellular enthesis with physical processes and chemical detergents. However, these methods did not achieve largesize enthesis decellularization with minimal disruption of the structure, composition, and mechanics of enthesis ECM. In this study, a novel VAS was developed for acquiring large-size AEM with similarity to native enthesis in structure, composition, and mechanics. An in vitro study determined that the O-AEM showed low immunogenicity without toxicity and hBMSCs cultured on the BR, FR, and TRs of the O-AEM spatially expressed the osteogenic, chondrogenic, and tenogenic genes, respectively. Additionally, enthesis regeneration was enhanced in vivo, as demonstrated by improved histological parameters in the O-AEM group. Overall, this new protocol may be helpful for the fabrication of a large-size AEM with good physicochemical and biological properties. This AEM may have potential clinical applications in enthesis tissueengineering.

Histologically, native enthesis comprises four transitional and gradient tissues: fibrous connective tendon, uncalcified fibrocartilage, calcified fibrocartilage, and bone $(1-3,6)$. The uncalcified and calcified fibrocartilages are avascular tissues with a dense, compact, and low-porosity architecture. Moreover, the fibrocartilage contains significantly more cells than the tendon and bone regions $(35,36)$. Therefore, the greatest challenge for large-size enthesis decellularization was to remove cells completely from the fibrocartilaginous zone without destroying its native ECM and inherent bioactive substances. Multiple freezethaw processes were utilized to fabricate various types of acellular scaffolds, including nerve, dermis, cartilage, and tendon (37-41). These previous studies indicated that freeze-thaw processing was a mild acellular treatment that could only lyse cells within tissues and cannot remove the lytic membranous and intracellular contents $(41,42)$.
Thus, we first repeated freeze-thaw processing five times to treat native enthesis. According to prior studies in the literature, chemical decellularization is a second step commonly used for decellularization after multiple freezethaw processes $(20,25,38)$. Chemicals frequently used for removing cell components include SDS and Triton X-100, while DNases and RNases are commonly used to remove nucleic acids from a tissue $(21,24)$. SDS, employed as an anionic surfactant, has the ability to lyse cells and denature proteins through disrupting non covalent bonds. This was successfully applied in decellularizing tendons, meniscus, cartilages, and bones (20-22,38). Triton X-100, employed as a nonionic surfactant, could permeabilize cellular membranes, solubilize membrane proteins, and extract DNA. Decellularization with Triton X-100 completely removed cellular components in tendons, nerves, and bones (43-45). Recently, Su et al. decellularized largesize enthesis using SDS and Triton X-100 followed by nuclear digestion (25). The AEM fabricated with this method preserved the natural structure and mechanical properties of native enthesis, but a significant fraction of collagen and GAGs in the fibrocartilage layer were washed away (25). The decellularization effect of the surfactants depends on the concentrations of detergents and the decellularization time (46). Tissues exposed to these agents with high concentration and/or long time could improve acellular efficiency, but they will wash away the inherent bioactive substances and inevitably denature the ECM proteins by disrupting non covalent bonds, thus altering the mechanical properties of the ECM $(26,27)$. Based on this phenomenon, we speculate that the loss of collagen and GAGs in this AEM may be attributed to the extensive treatment of surfactants with high concentration. To improve this aspect, we avoided the usage of surfactants in enthesis decellularization. More recently, Liu et al. developed an acellular protocol for canine patellar tendon enthesis without using detergents (31), but this protocol is still complicated and time-inefficient. Concerning large-size enthesis with thick fibrocartilage layer, the time required for decellularization may be even longer. Herein, we set up a VAS to create a sub-atmospheric pressure environment. Then, we decellularized the large-size enthesis in it without chemical surfactants. This method significantly shortened the time for decellularization and properly preserved the composition, structure, and tensile properties of native enthesis. Therefore, we consider that the vacuum aspiration described in this study may be a promising physical method for compact tissue decellularization. 
$\mathrm{H} \& \mathrm{E}$ and DAPI staining determined that the cellular components and nuclear substances were completely removed in the O-AEM. The DNA content in the O-AEM was quantitatively reduced to an extremely low level. Apart from removing cell components, another essential requirement was to preserve the ECM components. Considering that collagen, GAGs, minerals, and inherent GFs were the main components of enthesis ECM, they constituted the components evaluated in this study. Our results show that both $\mathrm{C}-\mathrm{AEM}$ and $\mathrm{O}-\mathrm{AEM}$ presented a similar distribution of collagen and GAGs with native enthesis, but the preservation of collagen and GAGs in the $\mathrm{O}-\mathrm{AEM}$ was better than in the C-AEM. Since the modulus ranges drastically across BTI $(47,48)$ to match the moduli of the stiff, ideal decellularized protocol should preserve the graded mineral distribution of NET. Calcium and phosphorus are the two essential elements for the initial generation of hydroxyapatite (HA), which is widely known to participate in the biomineralization process through its deposition in the ECM of hard tissue (49). Thus, the contents of the two elements were used as a parameter to describe the mineral level in AEM. EDS analysis confirmed that the $\mathrm{Ca}$ and $\mathrm{P}$ contents near the calcified fibrocartilage and bone were properly preserved, which may be one of the reasons for the osteogenic differentiation of interacted stem cells and good tensile resistance (50). ELISA results indicate that the GFs in the O-AEM were preserved much better than in the C-AEM. In particular, a greater amount of BMP-2, TGF- $\beta 1$, and CTGF was respectively found in the BR, FR, and TRs of the O-AEM. This may be the main reason why the stem cells on the O-AEM spatially down osteogenic, chondrogenic and tenogenic lineages better than the C-AEM.

Given that the surface topography of scaffolds directly affects cell attachment, proliferation, and differentiation (51-53), SEM was used to evaluate the surface topography of the NET, C-AEM, and O-AEM comparatively. Our results show that both O-AEM and C-AEM exhibited a similar surface topography with native enthesis. This may be the other reason why the triphasic scaffold enables hBMSCs to achieve regional differentiation. In addition, the porosity and water absorption of the C-AEM and O-AEM were significantly increased compared to native enthesis, which may be beneficial for endogenous cell attachment and migration as well as in vivo body fluid infiltration.

Taking into account that the function of enthesis was to mediate and translate the tensile loading from muscle contraction $(1,2,23)$, we can infer that an ideal AEM should also have good tensile resistance. Thus, it could replace the injured enthesis to resist tensile loading at the early stage of in vivo implantation. In this study, the stiffness of O-AEM was reduced, which may give rise to side effects of decellularization. Remarkably, O-AEM properly preserved the failure load of native enthesis, which may have a positive function for the graft to mediate and translate tensile loading at an early stage of RC healing. Additionally, all O-AEM samples were ruptured in the middle part of the tendon during the mechanical test. This was in agreement with the tensile properties of enthesis, showing that the role of the FR of the O-AEM for alleviating the tensile concentration was at least partially preserved (54).

The limitations of this study are described as follows: (I) only a histological evaluation of the regenerative capability of the O-AEM was carried out using a canine model; mechanical behavior and even motor ability should be determined in future studies; (II) at postoperative week 8 , the regenerated IT enthesis in the O-AEM group showed better histological features than those of the C-AEM group, but the underlying mechanisms were not elucidated. According to prior studies (55), investigation of specific mechanisms from the protein profiles of the AEM using a mass spectrometric procedure may be possible. The next step will be to investigate this possibility. Despite the aforementioned limitations, the optimized protocol proposed in this study provides an effective way for largesize enthesis decellularization. As a novel highly biomimetic scaffold, the fabricated O-AEM may be a promising graft for patching large/massive $\mathrm{RC}$ tear in clinic.

\section{Conclusions}

In this study, we set up a custom-designed VAS to decellularize large-size enthesis tissue, that avoids the use of detergents and saves a notable amount of time. According to a comparative analysis, this O-AEM preserved the morphologies, ingredients, and tensile properties much better than the C-AEM. Additionally, in vitro studies demonstrated that the C-AEM and O-AEM exhibit no cytotoxicity and low immunogenicity, allowing cell attachment and proliferation. The O-AEM also showed better region-specific inducibility on the interacted stem cell down osteogenic, chondrogenic, and tenogenic lineages than the C-AEM. In vivo studies indicate that this O-AEM could histologically accelerate enthesis regeneration in a canine IT repair model. This optimized protocol may be used to decellularize large-size enthesis efficiently as 
scaffolds for patching large/massive RC tear in clinic.

\section{Acknowledgments}

The authors would like to thank the staff of BL01B beamline at National Center for Protein Sciences Shanghai and Shanghai Synchrotron Radiation Facility, China, for assistance during SR-FTIR data collection.

Funding: This work was supported by the National Natural Science Foundation of China (No. 81730068 and 81902192), the China Postdoctoral Science Foundation (No. 2019M652809) and the Fundamental Research Funds for the Central Universities of Central South University (No. 2019zzts900 and 2019zzts350).

\section{Footnote}

Reporting Checklist: The authors have completed the ARRIVE reporting checklist. Available at http://dx.doi. org/10.21037/atm-20-3661

Data Sharing Statement: Available at http://dx.doi. org/10.21037/atm-20-3661

Peer Review File: Available at http://dx.doi.org/10.21037/ atm-20-3661

Conflicts of Interest: All authors have completed the ICMJE uniform disclosure form (available at http://dx.doi. org/10.21037/atm-20-3661). The authors have no conflicts of interest to declare.

Ethics Statement: The authors are accountable for all aspects of the work in ensuring that questions related to the accuracy or integrity of any part of the work are appropriately investigated and resolved. All procedures complied with the Animals (Scientific Procedures) Act 1986 and received university ethical approval of Xiangya Hospital, Central South University (No. 2019030517).

Open Access Statement: This is an Open Access article distributed in accordance with the Creative Commons Attribution-NonCommercial-NoDerivs 4.0 International License (CC BY-NC-ND 4.0), which permits the noncommercial replication and distribution of the article with the strict proviso that no changes or edits are made and the original work is properly cited (including links to both the formal publication through the relevant DOI and the license).
See: https://creativecommons.org/licenses/by-nc-nd/4.0/.

\section{References}

1. Atesok K, Fu FH, Wolf MR, et al. Augmentation of tendon-to-bone healing. J Bone Joint Surg Am 2014;96:513-21.

2. Derwin KA, Galatz LM, Ratcliffe A, et al. Enthesis repair: challenges and opportunities for effective tendon-to-bone healing. J Bone Joint Surg Am 2018;100:e109.

3. Rossetti L, Kuntz LA, Kunold E, et al. The microstructure and micromechanics of the tendon-bone insertion. Nat Mater 2017;16:664-70.

4. Lu HH, Thomopoulos S. Functional attachment of soft tissues to bone: development, healing, and tissue engineering. Annu Rev Biomed Eng 2013;15:201-26.

5. Lu H, Chen $\mathrm{C}, \mathrm{Qu} \mathrm{J}$, et al. Initiation timing of lowintensity pulsed ultrasound stimulation for tendonbone healing in a rabbit model. Am J Sports Med 2016;44:2706-15.

6. Genin GM, Kent A, Birman V, et al. Functional grading of mineral and collagen in the attachment of tendon to bone. Biophys J 2009;97:976-85.

7. Zelzer E, Blitz E, Killian ML, et al. Tendon-to-bone attachment: from development to maturity. Birth Defects Res C Embryo Today 2014;102:101-12.

8. Benjamin M, Toumi H, Ralphs JR, et al. Where tendons and ligaments meet bone: attachment sites ('entheses') in relation to exercise and/or mechanical load. J Anat 2006;208:471-90.

9. Galatz LM, Ball CM, Teefey SA, et al. The outcome and repair integrity of completely arthroscopically repaired large and massive rotator cuff tears. J Bone Joint Surg Am 2004;86:219-24.

10. Al-Hakim W, Noorani A, Lambert S. Assessment and treatment strategies for rotator cuff tears. Shoulder Elbow 2015;7:76-84.

11. Harada $Y$, Mifune $Y$, Inui A, et al. Rotator cuff repair using cell sheets derived from human rotator cuff in a rat model. J Orthop Res 2017;35:289-96.

12. Deprés-Tremblay G, Chevrier A, Snow M, et al. Rotator cuff repair: a review of surgical techniques, animal models, and new technologies under development. J Shoulder Elbow Surg 2016;25:2078-85.

13. Zheng C, Lu H, Tang Y, et al. Autologous freeze-dried, platelet-rich plasma carrying icariin enhances bonetendon healing in a rabbit model. Am J Sports Med 2019;47:1964-74. 
14. Hu J, Qu J, Xu D, et al. Combined application of lowintensity pulsed ultrasound and functional electrical stimulation accelerates bone-tendon junction healing in a rabbit model. J Orthop Res 2014;32:204-9.

15. Spalazzi JP, Dagher E, Doty SB, et al. In vivo evaluation of a multiphased scaffold designed for orthopaedic interface tissue engineering and soft tissue-to-bone integration. J Biomed Mater Res A 2008;86:1-12.

16. Spalazzi JP, Dagher E, Doty SB, et al. In vivo evaluation of a tri-phasic composite scaffold for anterior cruciate ligament-to-bone integration. Conf Proc IEEE Eng Med Biol Soc 2006;2006:525-8.

17. Phillips JE, Burns KL, Le Doux JM, et al. Engineering graded tissue interfaces. Proc Natl Acad Sci U S A 2008; 105:12170-5.

18. Wang IE, Shan J, Choi R, et al. Role of osteoblastfibroblast interactions in the formation of the ligament-tobone interface. J Orthop Res 2007;25:1609-20.

19. Paxton JZ, Donnelly K, Keatch RP, et al. Engineering the bone-ligament interface using polyethylene glycol diacrylate incorporated with hydroxyapatite. Tissue Eng Part A 2009;15:1201-9.

20. Chen C, Liu F, Tang Y, et al. Book-shaped acellular fibrocartilage scaffold with cell-loading capability and chondrogenic inducibility for tissue-engineered fibrocartilage and bone-tendon healing. ACS Appl Mater Interfaces 2019;11:2891-907.

21. Cheng CW, Solorio LD, Alsberg E. Decellularized tissue and cell-derived extracellular matrices as scaffolds for orthopaedic tissue engineering. Biotechnol Adv 2014;32:462-84.

22. Jakus AE, Laronda MM, Rashedi AS, et al. "Tissue papers" from organ-specific decellularized extracellular matrices. Adv Funct Mater 2017;27:1700992.

23. Benjamin M, McGonagle D. Entheses: tendon and ligament attachment sites. Scand J Med Sci Sports 2009;19:520-7.

24. Blaudez F, Ivanovski S, Hamlet S, et al. An overview of decellularisation techniques of native tissues and tissue engineered products for bone, ligament and tendon regeneration. Methods 2020;171:28-40.

25. Su M, Zhang Q, Zhu Y, et al. Preparation of decellularized triphasic hierarchical bone-fibrocartilage-tendon composite extracellular matrix for enthesis regeneration. Adv Healthc Mater 2019;8:e1900831.

26. Moriyama Y, Watanabe E, Kobayashi K, et al. Secondary structural change of bovine serum albumin in thermal denaturation up to 130 degrees $\mathrm{C}$ and protective effect of sodium dodecyl sulfate on the change. J Phys Chem B 2008;112:16585-9.

27. Xu K, Kuntz LA, Foehr P, et al. Efficient decellularization for tissue engineering of the tendon-bone interface with preservation of biomechanics. PLoS One 2017;12:e0171577.

28. Burk J, Erbe I, Berner D, et al. Freeze-thaw cycles enhance decellularization of large tendons. Tissue Eng Part C Methods 2014;20:276-84.

29. Bronstein JA, Woon CY, Farnebo S, et al. Physicochemical decellularization of composite flexor tendon-bone interface grafts. Plast Reconstr Surg 2013;132:94-102.

30. Burgkart R, Tron A, Prodinger P, et al. Decellularized kidney matrix for perfused bone engineering. Tissue Eng Part C Methods 2014;20:553-61.

31. Liu Q, Hatta T, Qi J, et al. Novel engineered tendonfibrocartilage-bone composite with cyclic tension for rotator cuff repair. J Tissue Eng Regen Med 2018;12:1690-701.

32. Ide J, Kikukawa K, Hirose J, et al. The effect of a local application of fibroblast growth factor-2 on tendon-tobone remodeling in rats with acute injury and repair of the supraspinatus tendon. J Shoulder Elbow Surg 2009;18:391-8.

33. Shah SA, Kormpakis I, Havlioglu N, et al. Sclerostin antibody treatment enhances rotator cuff tendon-tobone healing in an animal model. J Bone Joint Surg Am 2017;99:855-64.

34. Liu Q, Yu Y, Reisdorf RL, et al. Engineered tendonfibrocartilage-bone composite and bone marrow-derived mesenchymal stem cell sheet augmentation promotes rotator cuff healing in a non-weight-bearing canine model. Biomaterials 2019;192:189-98.

35. Kang H, Peng J, Lu S, et al. In vivo cartilage repair using adipose-derived stem cell-loaded decellularized cartilage ECM scaffolds. J Tissue Eng Regen Med 2014;8:442-53.

36. Gong YY, Xue JX, Zhang WJ, et al. A sandwich model for engineering cartilage with acellular cartilage sheets and chondrocytes. Biomaterials 2011;32:2265-73.

37. Guo L, Qu J, Zheng C, et al. Preparation and characterization of a novel decellularized fibrocartilage "book" scaffold for use in tissue engineering. PLoS One 2015;10:e0144240.

38. Xie S, Zhou Y, Tang Y, et al. -Book-shaped decellularized tendon matrix scaffold combined with bone marrow mesenchymal stem cells-sheets for repair of achilles tendon defect in rabbit. J Orthop Res 2019;37:887-97.

39. Gulati AK. Evaluation of acellular and cellular nerve 
grafts in repair of rat peripheral nerve. J Neurosurg 1988;68:117-23.

40. Bullard KM, Banda MJ, Arbeit JM, et al. Human acellular dermal matrix as a novel model of malignant epithelial cell invasion. Invasion Metastasis 1997;17:42-52.

41. Ning LJ, Zhang Y, Chen XH, et al. Preparation and characterization of decellularized tendon slices for tendon tissue engineering. J Biomed Mater Res A 2012;100:1448-56.

42. Gilbert TW, Sellaro TL, Badylak SF. Decellularization of tissues and organs. Biomaterials 2006;27:3675-83.

43. Whitlock PW, Smith TL, Poehling GG, et al. A naturally derived, cytocompatible, and architecturally optimized scaffold for tendon and ligament regeneration. Biomaterials 2007;28:4321-9.

44. Dong SW, Ying DJ, Duan XJ, et al. Bone regeneration using an acellular extracellular matrix and bone marrow mesenchymal stem cells expressing Cbfa1. Biosci Biotechnol Biochem 2009;73:2226-33.

45. Sun XH, Che YQ, Tong XJ, et al. Improving nerve regeneration of acellular nerve allografts seeded with SCs bridging the sciatic nerve defects of rat. Cell Mol Neurobiol 2009;29:347-53.

46. Elder BD, Eleswarapu SV, Athanasiou KA. Extraction techniques for the decellularization of tissue engineered articular cartilage constructs. Biomaterials 2009;30:3749-56.

47. Boys AJ, McCorry MC, Rodeo S, et al. Next generation tissue engineering of orthopedic soft tissue-to-bone interfaces. MRS Commun 2017;7:289-308.

48. Liu Y, Thomopoulos S, Chen C, et al. Modelling the mechanics of partially mineralized collagen fibrils, fibres

Cite this article as: Shi Q, Chen Y, Li M, Zhang T, Ding S, $\mathrm{Xu} \mathrm{Y,} \mathrm{Hu} \mathrm{J,} \mathrm{Chen} \mathrm{C,} \mathrm{Lu} \mathrm{H.} \mathrm{Designing} \mathrm{a} \mathrm{novel} \mathrm{vacuum}$ aspiration system to decellularize large-size enthesis with preservation of physicochemical and biological properties. Ann Transl Med 2020;8(21):1364. doi: 10.21037/atm-20-3661 and tissue. J R Soc Interface 2013;11:20130835.

49. Lu H, Chen C, Wang Z, et al. Characterization of calcium and zinc spatial distributions at the fibrocartilage zone of bone-tendon junction by synchrotron radiationbased micro X-ray fluorescence analysis combined with backscattered electron imaging. Spectrochimica Acta Part B: Atomic Spectroscopy 2015;111:15-22.

50. Müller P, Bulnheim U, Diener A, et al. Calcium phosphate surfaces promote osteogenic differentiation of mesenchymal stem cells. J Cell Mol Med 2008;12:281-91.

51. Czeisler C, Short A, Nelson T, et al. Surface topography during neural stem cell differentiation regulates cell migration and cell morphology. J Comp Neurol 2016;524:3485-502.

52. Abagnale G, Sechi A, Steger M, et al. Surface topography guides morphology and spatial patterning of induced pluripotent stem cell colonies. Stem Cell Reports 2017;9:654-66.

53. Goriainov V, Cook RB, Murray JW, et al. Human skeletal stem cell response to multiscale topography induced by large area electron beam irradiation surface treatment. Front Bioeng Biotechnol 2018;6:91.

54. Wren TA, Yerby SA, Beaupre GS, et al. Mechanical properties of the human achilles tendon. Clin Biomech (Bristol, Avon) 2001;16:245-51.

55. Schneider KH, Enayati M, Grasl C, et al. Acellular vascular matrix grafts from human placenta chorion: impact of ECM preservation on graft characteristics, protein composition and in vivo performance. Biomaterials 2018;177:14-26. 